\title{
BMJ
}

\section{Effect of high throughput $R H D$ typing of fetal DNA in maternal plasma on use of anti-RhD immunoglobulin in RhD negative pregnant women: prospective feasibility study}

\author{
Kirstin Finning, clinical scientist, ${ }^{1}$ Pete Martin, clinical scientist, Joanna Summers, biomedical scientist, \\ Edwin Massey, consultant haematologist, ${ }^{1}$ Geoff Poole, head of red cell immunohaematology, ${ }^{2}$ Geoff \\ Daniels, head of molecular diagnostics ${ }^{1}$
}

\begin{abstract}
1 International Blood Group Reference Laboratory, NHS Blood and Transplant, Bristol BS10 5ND ${ }^{2}$ NHS Blood and Transplant, Bristol

Correspondence to: G Daniels geoff.daniels@nbs.nhs.uk
\end{abstract}

doi:10.1136/bmj.39518.463206.25

\section{ABSTRACT}

Objectives To assess the feasibility of applying a high throughput method, with an automatic robotic technique, for predicting fetal RhD phenotype from fetal DNA in the plasma of RhD negative pregnant women to avoid unnecessary treatment with anti-RhD immunoglobulin. Design Prospective comparison of fetal RHD genotype determined from fetal DNA in maternal plasma with the serologically determined fetal RhD phenotype from cord blood.

Setting Antenatal clinics and antenatal testing laboratories in the Midlands and north of England and an international blood group reference laboratory.

Participants Pregnant women of known gestation identified as RhD negative by an antenatal testing laboratory. Samples from 1997 women were taken at or before the 28 week antenatal visit.

Main outcome measures Detection rate of fetal RhD from maternal plasma, error rate, false positive rate, and the odds of being affected given a positive result.

Results Serologically determined RhD phenotypes were obtained from 1869 cord blood samples. In 95.7\% $(n=1788)$ the correct fetal RhD phenotype was predicted by the genotyping tests. In $3.4 \%(n=64)$ results were either unobtainable or inconclusive. A false positive result was obtained in $0.8 \%$ (14 samples), probably because of unexpressed or weakly expressed fetal $R H D$ genes. In only three samples $(0.2 \%)$ were false negative results obtained. If these results had been applied as a guide to treatment, only $2 \%$ of the women would have received anti-RhD unnecessarily, compared with $38 \%$ without the genotyping.

Conclusions High throughput RHD genotyping of fetuses in all RhD negative women is feasible and would substantially reduce unnecessary administration of anti$\mathrm{RhD}$ immunoglobulin to RhD negative pregnant women with an RhD negative fetus.

\section{INTRODUCTION}

Alloimmunisation against the $\mathrm{RhD}(\mathrm{RH} 1)$ red cell surface antigen is the commonest cause of haemolytic disease of the fetus and newborn, which, before the introduction of anti-D prophylaxis after delivery in the 1960 s accounted for the death of one baby in $2200 .^{12}$ Over the next 40 years the effect of the anti-RhD prophylaxis programme and improved neonatal care reduced the incidence to one death in $21000 .^{3}$

In 2002 the National Institute for Health and Clinical Excellence (NICE) in the United Kingdom undertook an assessment of the cost effectiveness of routine antenatal anti-RhD prophylaxis with anti-RhD immunoglobulin. ${ }^{3}$ Previously anti-RhD immunoglobulin had been administered antenatally only when events occurred that would be associated with a fetomaternal haemorrhage. NICE recommended that all RhD negative pregnant women should be offered anti$\mathrm{RhD}$ immunoglobulin at 28 and 34 weeks' gestation. ${ }^{3}$ In a predominantly white population, however, about $38 \%$ of these women would be carrying an $\mathrm{RhD}$ negative fetus ${ }^{4}$ and would receive the treatment unnecessarily. Consequently, NICE also "endorsed studies into the feasibility of mass testing antenatally for fetal blood group by analysis of fetal DNA in maternal plasma."3 The benefits of this testing would be twofold. Firstly, there would be a substantial reduction in the use of anti-RhD immunoglobulin, an expensive blood product in short supply. Secondly, women with an RhD negative fetus would be spared unnecessary exposure to this pooled human blood product with its associated discomfort and perceived risk from viral or prion contamination. This risk is exemplified by the infection of hundreds of women with hepatitis $\mathrm{C}$ virus transmitted by anti-RhD immunoglobulin in Ireland in 1977-8. ${ }^{5}$

Anti-RhD immunoglobulin is produced by the pooling and fractionation of plasma from large numbers of donors who themselves are $\mathrm{RhD}$ negative and have been exposed to $\mathrm{RhD}$ positive red cells to stimulate the production of $\mathrm{RhD}$ antibodies. Although the future of anti-RhD immunoglobulin might involve monoclonal or recombinant products, thus eliminating the risks associated with human blood products, there is still no indication that these are to be introduced in 
the foreseeable future and costs would probably increase if recombinant products were used. ${ }^{6}$

The antigens of the $\mathrm{Rh}$ blood group system are located on two proteins encoded by two homologous genes, $\mathrm{RHD}$ and $\mathrm{RHCE}^{4}$ The most immunogenic of the Rh antigens, RhD, is encoded by RHD. About $15 \%$ of white people are $\mathrm{RhD}$ negative and are usually homozygous for a deletion of $R H D$, whereas $\mathrm{RhD}$ positive people have either one or two copies of $R H D{ }^{7}$ Tests for predicting $\mathrm{RhD}$ phenotype from DNA involve the amplification of one or more regions of $R H D$ to determine whether the gene is present. Numerous variants of $R H D$ exist: in some, all or part of $R H D$ is present but no $\mathrm{RhD}$ antigen is expressed; in others, part of $R H D$ is absent but a variant form of $\mathrm{RhD}$ antigen is present. ${ }^{48} R H D$ variants are relatively rare in white people, but an inactive $R H D$ gene, called $R H D \Psi$, is present in $66 \%$ of $\mathrm{RhD}$ negative black Africans. ${ }^{9}$ $R H D \Psi$ contains a 37 base pair duplication plus a nonsense mutation and must be taken into account in any method for $R H D$ genotyping.

Since 1995 the International Blood Group Reference Laboratory of the English National Blood Service has been providing a fetal $R H D$ genotyping service for $\mathrm{RhD}$ alloimmunised women whose fetuses might be at risk of haemolytic disease of the fetus and newborn. ${ }^{1011}$ In 1997 Lo et al showed that about 3-6\% of cell-free DNA in the plasma of pregnant women is of fetal origin and can be used for predicting $\mathrm{RhD}$ fetal phenotype in RhD negative women. ${ }^{1213}$ In 2001 the laboratory introduced fetal $R H D$ genotyping from fetal DNA in maternal plasma to their molecular diagnostics service. Maternal plasma has now almost replaced fetal cells, obtained by amniocentesis or chorionic villus sampling, as the source of fetal DNA, hence eliminating the need for invasive sampling procedures. ${ }^{11}$ The method currently used routinely for fetal $R H D$ genotyping is labour intensive and expensive and therefore not suitable for the mass screening of all $\mathrm{RhD}$ negative women. Recent developments in technology and the introduction of automated robotic techniques have brought down costs and increased the potential for higher throughput.

We validated a high throughput $R H D$ fetal genotyping technique by comparing the results obtained with the $\mathrm{RhD}$ serological phenotype obtained from cord blood taken at delivery.

\section{METHODS}

Blood samples

Anticoagulated blood samples were chosen for fetal genotyping from any $\mathrm{RhD}$ negative pregnant women attending antenatal clinics that use the Birmingham and Sheffield centres of the National Blood Service for routine $\mathrm{ABO}$ and $\mathrm{RhD}$ blood grouping and antibody screening. This did not involve taking additional blood samples to those collected for routine testing. The blood samples were collected at the women's 28 week visit to the antenatal clinic. Ethnicity was 55\% white, 8\% Asian, 1.5\% black, 0.5\% Caribbean black, 1\% mixed, $1 \%$ other, and $33 \%$ unknown or not given. We selected at least 1500 individuals on the basis of $80 \%$ power to detect an error rate of $2 \%$.

\section{Robotic isolation of DNA from maternal plasma}

Bar coded blood tubes containing $4-6 \mathrm{ml}$ of anticoagulated blood were centrifuged for 10 minutes and the tubes were then transferred directly to the sample rack of the MDx BioRobot (Qiagen, Hilden, Germany) without disturbing the buffy coat. DNA was robotically extracted from $0.56 \mathrm{ml}$ of maternal plasma and eluted into nuclease-free water in a 96 well plate. This procedure took about three hours, with a capacity of 88 blood samples per run. The extracted DNA was used for real time quantitative polymerase chain reaction immediately or stored at $-30^{\circ} \mathrm{C}$ until testing was performed.

Real time quantitative polymerase chain reaction to detect the presence of $R H D$

The MDx BioRobot was used as a liquid handling robot to dispense a mix of Universal PCR MasterMix (Applied Biosystems, Foster City, CA, USA) and primers and probes for exons 5 and 7 of the $R H D$ gene (as a multiplex) or the $C C R 5$ gene (see table A on bmj.com) into a 384 well polymerase chain reaction plate. DNA $(5 \mu \mathrm{l})$ from each maternal sample was dispensed into three wells containing reaction mix for the $R H D$ gene and into a single well containing reaction mix for CCR5. Control wells for the $R H D$ assay containing RhD positive DNA, RhD negative DNA, $R H D \Psi$ positive DNA, and no DNA were also dispensed. Real time quantitative polymerase chain reactions were performed on the ABI Prism 7900HT (Applied Biosystems) to detect the presence of fetal $R H D$ gene sequences and to quantify total (maternal and fetal) DNA in the plasma. These tests were interpreted without knowledge of the $\mathrm{RhD}$ phenotype, which was subsequently determined on red cells from the cord blood sample. The procedure took about three hours with a capacity of DNA from 88 blood samples per run.

Interpretation of real time polymerase chain reaction results

The $R H D$ exon 7 assay amplified both $R H D$ and $R H D \Psi$, whereas the exon 5 assay amplified $R H D$ only. A cycle threshold value of less than 42 was interpreted as a positive signal. An algorithm was created to predict fetal RhD phenotype and to recommend whether anti$\mathrm{RhD}$ should be offered or withheld, depending on the number of positive signals obtained for both $R H D$ exons 5 and 7. Confirmation of successful DNA extraction and an estimation of the amount of total DNA in the sample were provided by the single amplification of $C C R 5$ (not fetal specific).

\section{Statistical analysis}

We used GraphPad Prism 5 software package (GraphPad Software, San Diego, CA) to analyse the results. We used Fisher's exact test (two tailed) to determine the 
sensitivity and specificity of the test and the MannWhitney rank sum test to compare the effect of total DNA concentration on the accuracy of the prediction of fetal $\mathrm{RhD}$ status and whether to recommend giving anti-RhD immunoglobulin. We regarded a two tailed probability value ( $\mathrm{P}$ value) of $<0.05$ as significant.

\section{Serological RhD testing on cord bloods}

The accredited testing laboratories in the National Blood Service and in hospital trusts performed $\mathrm{RhD}$ typing on cord blood samples using routine serological methods. At that time they did not know the genotype as determined from the fetal DNA.

\section{RESULTS}

\section{Paired analyses}

RHD genotyping was performed on DNA from 1997 pregnant women, with a mean gestational age of 27 . 9 weeks (median 28 weeks, range 8 -38 weeks). Most $(92 \%)$ samples were tested at 26-32 weeks' gestation. Cord blood RhD phenotypes were available from 1869 deliveries; 128 fetal phenotypes were not available for paired analysis because 124 cord samples were untraceable and there were four fetal deaths. When twins were delivered $(n=13)$, the expected genotype from maternal plasma was $R H D$ positive if at least one of the babies was $\mathrm{RhD}$ positive.

\section{Prediction of fetal RhD phenotype from DNA}

In $95.7 \%$ of cases the fetal $\mathrm{RhD}$ status predicted from the genotype was the same as the serologically determined phenotype from cord blood: 1118 $(59.8 \%)$ were $\mathrm{RhD}$ positive and $670(35.9 \%)$ were $\mathrm{RhD}$ negative (table). There were 14 false positive results $(0.8 \%)$, in which an $R H D$ gene was detected but the phenotype determined serologically from cord blood was RhD negative. Cord blood samples were not available for DNA extraction so we were unable to test for the presence of variants of $R H D$ associated with non-expression or weak expression of the $\mathrm{RhD}$ antigen, the most likely and expected causes of these apparently incorrect results. Only three samples $(0.16 \%)$ gave "false negative" results. Eight samples

Results of testing 1869 DNA samples from plasma of RhD negative pregnant women for fetal $R H D$ and comparison with serologically determined phenotype of her baby's cord sample*

\begin{tabular}{|c|c|c|c|}
\hline $\begin{array}{l}\text { Predicted phenotype } \\
\text { from fetal DNA }\end{array}$ & $\begin{array}{l}\text { Serological phenotype of cord } \\
\text { sample }\end{array}$ & No (\%) & Conclusion \\
\hline RhD postive & RhD postive & $1118(59.8)$ & Correct \\
\hline RhD negative & RhD negative & $670(35.9)$ & Correct \\
\hline RhD positive & RhD negative & $14(0.8)$ & False positive \\
\hline RhD negative & RhD postive & $3(0.2)$ & False negative \\
\hline$R H D$ variant & 4 RhD postive/4 RhD negative & $8(0.4)$ & Inconclusive \\
\hline Inconclusive & $13 \mathrm{RhD}$ postive $\mathrm{f} / 18 \mathrm{RhD}$ negative & $31(1.7)$ & Inconclusive \\
\hline Inconclusiveł & $18 \mathrm{RhD}$ postive/7 RhD negative & $25(1.3)$ & Inconclusive \\
\hline \multicolumn{4}{|c|}{$\begin{array}{l}\text { * If anti-RhD is given only when predicted phenotype from DNA is RhD positive, sensitivity of test is } 96.7 \% \text { ( } 95 \% \\
\text { Cl } 95.5 \% \text { to } 97.6 \%) \text { and specificity is } 98 \%(96.7 \% \text { to } 98.8 \%) \text {. If anti-RhD is given when predicted phenotype } \\
\text { from DNA is RhD positive, RHD variant, or inconclusive, sensitivity is } 99.7 \%(99.2 \% \text { to } 99.9 \%) \text { and specificity is } \\
94 \% \text { ( } 92.0 \% \text { to } 95.5 \%) \text {. } \\
\text { †One resulting from DNA extraction failure. } \\
\ddagger R H D \text { detected in maternal DNA. }\end{array}$} \\
\hline
\end{tabular}

$(0.4 \%)$ gave "variant $R H D$ " results, which were exon 7 positive but exon 5 negative. This pattern would be expected if the fetus had inherited $R H D \Psi$ or, less commonly, the variant RHDVI gene. Four of these eight fetuses were subsequently shown to be serologically RhD negative and might have had these variant genes, and the remaining four were $\mathrm{RhD}$ positive.

Inconclusive fetal genotyping results were obtained from 56 samples (3\%), of which 31 were from fetuses that were $\mathrm{RhD}$ positive and 25 were from fetuses that were $\mathrm{RhD}$ negative. One inconclusive result arose from a failure to extract DNA from the plasma. Thirty samples gave insufficient positive and negative replicates and so we categorised them as inconclusive. Twenty five of the inconclusive results (1.3\%) arose from the presence of a suspected maternal $R H D$ gene and in these cases the fetal phenotype was not predicted. Further tests on DNA derived from the women's buffy coats revealed that three women were normal $\mathrm{RhD}$ negative, indicating an excessively large amount of fetal DNA in their plasma. Ten women had $R H D \Psi$, one had RHDVI, and one had a single point mutation in $R H D(722 \mathrm{C}>\mathrm{T}$, Thr241Ile), apparently preventing full expression of the $\mathrm{RhD}$ protein. The remaining 10 women all had at least an RHD exon 7, but further analysis of their $R H$ genes was not performed.

Accuracy of test to determine requirement for anti-RhD administration

We compared two alternative models for determining the accuracy of the maternal plasma test. The fetus was categorised as "screen positive" if either the predicted phenotype from DNA was RhD positive, or the predicted phenotype from DNA was RhD positive, $R H D$ variant, or inconclusive. With the first model, the detection rate was $96.7 \%$, the false positive rate was $1.96 \%$, and the odds of being $\mathrm{RhD}$ positive given a positive result were $80: 1$. By the second method, the detection rate was $99.7 \%$, the false positive rate was $6.0 \%$, and the odds of being $\mathrm{RhD}$ positive given a positive result were $27: 1$. The second, more cautious, definition of "screen positive" is therefore more beneficial because more $\mathrm{RhD}$ positive fetuses are detected for an acceptable increase in the false positive rate (figure). If this model was applied to all $\mathrm{RhD}$ negative pregnant women in England and Wales, it would prevent a considerable number of women with $\mathrm{RhD}$ positive fetuses being omitted from treatment (figure). If it had been used to decide which women in this trial should receive prophylaxis, $64 \%$ would have received anti-RhD (of whom $96.4 \%$ had $\mathrm{RhD}$ positive babies). Of the women in the trial, $62 \%$ gave birth to $\mathrm{RhD}$ positive babies and therefore only $2 \%$ of women would have received anti-RhD unnecessarily, compared with $38 \%$ without genotyping.

\section{Discrepant results}

We analysed the effect of DNA concentration in maternal plasma on the ability to determine fetal $R H D$ genotype. We compared the amount of DNA in 
the three maternal plasma samples that gave false negative results and in the 31 inconclusive samples (where maternal $R H D$ was not present) with the amount of DNA in maternal plasma samples when the fetus was correctly predicted to be $\mathrm{RhD}$ positive. The mean total DNA concentration was found to be significantly higher in the false negative and inconclusive samples $(\mathrm{P}<0.001)$, which might explain the failure to amplify fetal DNA in these cases. Many samples that gave false negative or inconclusive results were more than 14 days old and had excessively high levels of maternal DNA, probably as a result of the breakdown of maternal leucocytes. Under usual clinical laboratory criteria these samples would not have been accepted for testing and repeat samples would have been requested.

\section{DISCUSSION}

We have developed a high throughput technology for determining fetal $R H D$ genotype from cell free fetal DNA in the plasma of RhD negative pregnant women. We tested almost 2000 samples from RhD negative women and compared the results with $\mathrm{RhD}$ phenotypes obtained by serological testing of red cells from the corresponding cord blood samples. There was concurrence between genotype and phenotype in $95.7 \%$ of the tests. In $0.8 \%$ of the tests the genotype falsely predicted an RhD positive phenotype and in $0.16 \%$ of the tests the genotype falsely predicted an $\mathrm{RhD}$ negative phenotype. In the remaining $3.4 \%$ of the tests the genotyping results were inconclusive.

\section{Benefits of fetal RhD testing}

In accordance with current NICE guidelines, ${ }^{3}$ all RhD negative women in the UK should be offered routine antenatal anti-RhD prophylaxis of at least $500 \mathrm{IU}$ of anti-RhD immunoglobulin at 28 and 34 weeks' gestation or a single dose of 1500 IU at 28 weeks' gestation. About $38 \%$ of white $\mathrm{RhD}$ negative women carry an $\mathrm{RhD}$ negative fetus and receive anti- $\mathrm{RhD}$

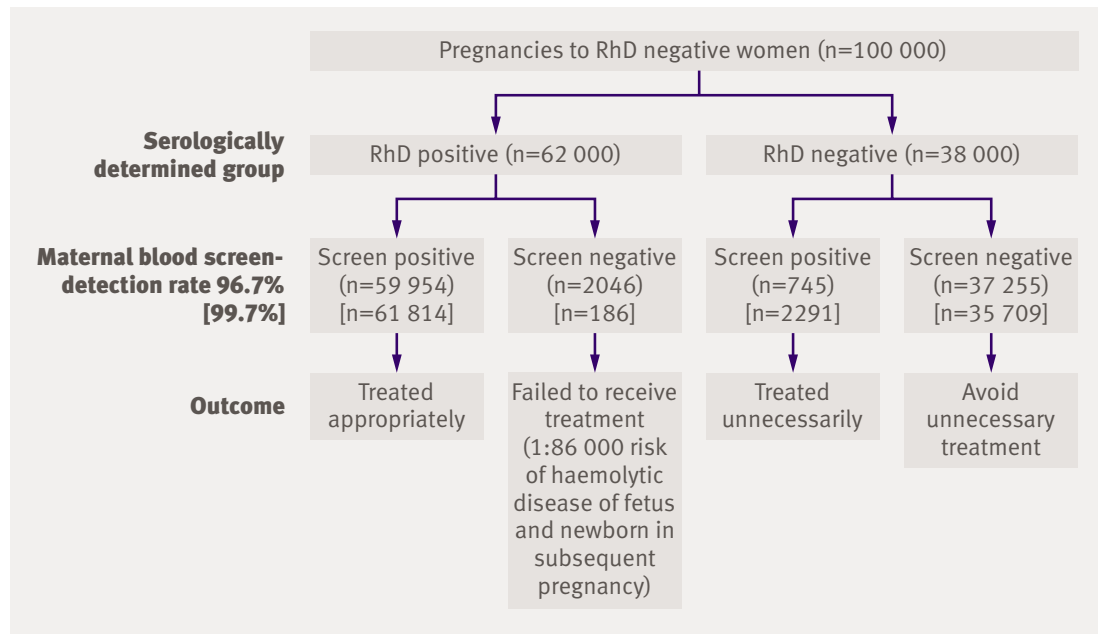

Hypothetical flow diagrams of fetal $R H D$ screening from maternal blood, with positive screen result classes as predicted phenotype from DNA of RhD positive only or with predicted phenotype from DNA of RhD positive, $R H D$ variant, and inconclusive results immunoglobulin unnecessarily. ${ }^{4}$ Lack of a safe and reliable technique for routine fetal $R H D$ genotyping with sufficiently high throughput has meant that to protect all $\mathrm{RhD}$ negative women, those with an $\mathrm{RhD}$ negative fetus also receive prophylaxis.

Our results show that fetuses of $\mathrm{RhD}$ negative women could be $R H D$ genotyped with an acceptable level of accuracy. Application of the testing protocol before 28 weeks' gestation, while still leading to anti$\mathrm{RhD}$ administration in $2 \%$ of mothers with $\mathrm{RhD}$ negative fetuses, would avoid unnecessary treatment in about $36 \%$ of $\mathrm{RhD}$ negative women and avert the associated discomfort, inconvenience, and risk of exposure to pooled donor blood products that such injections entail. In theory, $\mathrm{RhD}$ negative women would be saved an additional visit to the midwife for a second dose at 34 weeks' gestation, though routine care might require such a visit. Potential sensitising events, such as abdominal trauma or vaginal bleeding, occurring after 28 weeks' gestation would not require additional treatment with anti-RhD immunoglobulin in the $36 \%$ of cases shown to have an $\mathrm{RhD}$ negative fetus. In addition, should these tests prove completely reliable, the routine $\mathrm{RhD}$ serological testing of cord blood samples from neonates of $\mathrm{RhD}$ negative mothers could be eliminated.

\section{Relevance of false positive results}

The incidence of an $R H D$ positive genotype associated with an $\mathrm{RhD}$ negative phenotype $(0.8 \%)$ is consistent with the prevalence of $R H D$ genetic variants associated with lack of phenotypic expression of an $\mathrm{RhD}$ antigen found in other studies. ${ }^{1415}$ Such "false positives" would, however, be of limited importance. Only a few mothers would continue to receive anti-RhD immunoglobulin unnecessarily with $R H D$ genotyping compared with the large numbers currently treated in the absence of fetal genotyping. In fact, the red cells of some of these fetuses probably express small quantities of $\mathrm{RhD}$ antigen, not detected by routine serological testing, and so administration of anti-RhD could be considered appropriate.

\section{Relevance of false negative results}

A false negative result could be more important because withholding treatment from women carrying an $\mathrm{RhD}$ positive fetus would be associated with potential alloimmunisation and morbidity or mortality from haemolytic disease of the fetus and newborn in subsequent pregnancies. The risk of alloimmunisation occurring when anti-RhD prophylaxis is given only after delivery is about $1 \%$ compared with $0.3 \%$ if antenatal prophylaxis is also given. ${ }^{3}$ The overall risk of antenatal alloimmunisation depends on the likelihood of a subsequent pregnancy $(62 \%)$ and that the baby will be $\mathrm{RhD}$ positive $(71.5 \%$, assuming no change of partner). Based on the observed detection rate in this trial of $99.7 \%$, the risk of future pregnancies being affected by haemolytic disease of the fetus and newborn that would have been prevented by giving all RhD negative women anti-D at 28 and 34 weeks' 
gestation is 1:86000 RhD negative women. About 5\% of sensitisations result in fetal or neonatal death and another 5\% of infants might experience mild to severe developmental problems, therefore a false negative result would lead to about one additional case of fetal or neonatal loss or of developmental problems occurring per $860000 \mathrm{RhD}$ negative pregnant women. This modest apparent increase in risk might, however, be offset by an increased uptake of prophylaxis among mothers who have been correctly identified as carrying an $\mathrm{RhD}$ positive fetus.

In our study only three samples gave an apparently false $\mathrm{RhD}$ negative result. Some of the samples tested in this trial, including these three, were delayed in transport, which could reduce accuracy of the results. Strict implementation of a policy of testing only samples that were less than seven days old would increase the logistical problems of transportation over large geographic areas but would reduce the risk of false negative results.

\section{Variant $R H D$ genes}

Categorisation of a fetus predicted to carry a variant $R H D$ gene as "screen positive" might be an overcautious approach as these are probably fetuses with either an inactive $R H D \Psi$ gene or with an RHDVIgene, which produces a weak variant $\mathrm{D}$ antigen, and neither is likely to cause alloimmunisation of the mother. If this method were introduced for routine testing, further validation of the test might result in such variants being treated as $\mathrm{RhD}$ negative and antenatal immunoglobulin therapy being withheld.

When the mother is predicted to carry an $R H D$ gene, a cautious approach should be applied. In this study, $72 \%$ of women suspected of having $R H D$ genes gave birth to RhD positive babies and treating such inconclusive cases as "screen positive" would seem to be the most appropriate approach.

\section{Application of the technology}

No diagnostic test has 100\% sensitivity and specificity, including the serological testing that was used as the reference for determining the accuracy of the molecular tests in this study. The earlier in pregnancy that fetal DNA is tested the greater the risk that it will not be detected because cell-free fetal DNA concentrations rise as pregnancy progresses. ${ }^{16}$ Most of our tests were performed on blood taken at the 28 week visit, during which the first dose of anti-RhD immunoglobulin is usually given. For the fetal $\mathrm{RhD}$ testing to be completed and the results fed back to the antenatal clinics before this visit, blood samples would need to be taken at 26 weeks' gestation. It is unlikely that this would make a significant difference to the accuracy of the testing. In the trial, 183 samples were from women at 26 weeks' gestation or earlier and tests on these resulted in no false negative results. Alternatively, if the policy on routine antenatal prophylaxis were modestly changed to a single dose of anti-RhD immunoglobulin given at 30 weeks' gestation, then testing at 28 weeks would be suitable. Commencement of treatment at 30 rather than 28 weeks has previously been considered as an option in the UK, ${ }^{17}{ }^{18}$ and since 1998 the practice in Holland has been to give $1000 \mathrm{IU}$ of anti-RhD at 30 weeks' gestation. $^{19}$

\section{Other trials}

A similar fully automated trial has been performed in Amsterdam, in which 2359 samples taken at 30 weeks' gestation were tested for $R H D$ exon 7 after robotic isolation of DNA from maternal plasma and real time quantitative polymerase chain reaction technology. ${ }^{20}$ In 1257 of the cases in which molecular results could be compared with serological results, researchers obtained three false negative results and five false positive results, giving a diagnostic accuracy of $99.4 \%$.

\section{Future studies}

Clearly there could be benefits from taking maternal blood samples for fetal $R H D$ genotyping at earlier stages of the pregnancy than 28 weeks' gestation to coincide with existing planned antenatal visits. Consequently, we are about to start feasibility trials on testing maternal blood samples obtained during the first visit to the antenatal clinic, normally at 8-16 weeks' gestation, and at the visit for the anomaly scan at 20 weeks.

\section{Conclusions}

High throughput $R H D$ genotyping performed on free fetal DNA in maternal plasma at about 28 weeks' gestation reliably predicts fetal $\mathrm{RhD}$ phenotype with an acceptably low rate of false negative results. The introduction of fetal genotyping followed by the withholding of antenatal anti-RhD prophylaxis from mothers with an $\mathrm{RhD}$ negative fetus would result in about 36\%of women being saved from unnecessary exposure to human blood products, inconvenience, and discomfort

We thank the many pregnant women who took part in this study. We are also grateful to lan Skidmore, Craig Wilkes, Richard Mason, and other managers and staff in the red cell immunohaematology laboratories of the National Blood Service, the staff of the hospital trusts in the Birmingham, Sheffield, and Oxford regions, and Helen Williams, Maggie Pailing, and Sue Cotton of the National Blood Service Hospital liaison team for their advice. Michael Spratt, research statistician of the University of Bristol, provided

\section{WHAT IS ALREADY KNOWN ON THIS TOPIC}

Fetal RhD phenotype can be predicted from fetal DNA in the plasma of RhD negative pregnant women

About $38 \%$ of RhD negative pregnant women have an RhD negative fetus and might receive unnecessary antenatal anti-RhD immunoglobulin

\section{WHAT THIS STUDY ADDS}

An automated high throughput method developed for determining fetal RhD phenotype from fetal DNA in the plasma of RhD negative pregnant women is accurate

Screening of fetal $R H D$ in all RhD negative pregnant women is feasible 
valuable statistical input into the design of the study, and lan Franklin, Ann Gooch, and Frank Boulton assisted with the manuscript.

Contributors: All authors participated in the study design, study implementation, and interpretation of the results. GD is guarantor. Funding: KF, PM, JS, and GD were supported by the UK Department of Health. Some funding for consumables was provided by the European Union through the Special Non-Invasive Advances in Fetal and Neonatal Evaluation (SAFE) Network of Excellence.

Competing interests: None declared.

Ethical approval: Central office for research ethics committees in the UK. Provenance and peer review: Not commissioned; externally peer reviewed.

1 Walker W, Murray S, Russell JK. Stillbirth due to haemolytic disease of the newborn. J Obstet Gynaecol Br Emp 1957;44:573-81.

2 Kumar S, Regan F. Management of pregnancies with RhD alloimmunisation. BMJ 2005;330:1255-8.

3 National Institute for Clinical Excellence. Technology appraisal guidance 41. Guidance on the use of routine antenatal anti-D prophylaxis for RhD-negative women. London: NICE, 2002.

4 Daniels G. Human blood groups. 2nd ed. Oxford: Blackwell Science, 2002.

5 Kenny-Walsh E. Clinical outcomes after hepatitis $\mathrm{C}$ infection from contaminated anti-D immune globulin. New Engl J Med 1999;340:1228-33.

6 Kumpel BM. Efficacy of RhD monoclonal antibodies in clinical trials as replacement therapy for prophylactic anti-D immunoglobulin: more questions than answers. Vox Sang 2007;93:99-111.

7 Colin Y, Chérif-Zahar B, Le Van Kim C, Raynal V, Van Huffel V, Cartron J$\mathrm{P}$. Genetic basis of the RhD-positive and RhD-negative blood group polymorphism as determined by Southern analysis. Blood 1991;78:2747-52.

8 Westhoff CM. The structure and function of the Rh antigen complex. Semin Hematol 2007;44:42-50.
9 Singleton BK, Green CA, Avent ND, Martin PG, Smart E, Daka A, et al. The presence of an RHD pseudogene containing a 37 base pair duplication and a nonsense mutation in most Africans with the Rh D negative blood group phenotype. Blood 2000;95:12-8.

10 Finning KM, Martin PG, Soothill PW, Avent ND. Prediction of fetal D status from maternal plasma: introduction of a new noninvasive fetal RHD genotyping senvice. Transfusion 2002;42:1079-85.

11 Daniels G, Finning K, Martin P, Summers J. Fetal blood group genotyping. Present and future. Ann NY Acad Sci 2006;1075:88-95.

12 Lo YMD, Corbetta N, Chamberlain PF, Rai V, Sargent IL, Redman CWG. Presence of fetal DNA in maternal plasma and serum. Lancet 1997;350:485-7.

13 Lo YMD. Fetal RhD genotyping from maternal plasma. Ann Med 1999;31:308-12.

14 Wagner FF, Frohmajer A, Flegel WA. RHD positive haplotypes in D negative Europeans. BMC Genet 2001;2:10.

15 Denomme GA, Wagner FF, Fernandes BJ, Li W, Flegel WA. Partial D, weak D types, and novel RHD alleles among 33,864 multiethnic patients: implications for anti-D alloimmunization and prevention. Transfusion 2005;45:1554-60.

16 Lo YM, Tein MSC, Lau TK, Haines C), Leung TN, Poon PMK, et al. Quantitative analysis of fetal DNA in maternal plasma and serum: implications for noninvasive prenatal diagnosis. Am J Hum Genet 1998;62:768-75.

17 Lee D. Preventing RhD haemolytic disease of the newborn. BMJ 1998;316:1611.

18 Lee D. Proposed new UK recommendations. Br J Obstet Gynaecol 1998;105(s18):23.

19 De Haas M, van der Schoot E, Overbeeke M, Bonsel G, Vrijkotte T. International forum: current status of immunoprohylaxis with anti-D immunoglobulin. Vox Sang 2003;85:333-4.

20 Van der Schoot CE, Soussan AA, Koelewijn J, Bonsel GJ, PagetChristiaens LGC, de Haas M. Non-invasive antenatal RHD typing. Transfus Clin Biol 2006;13:53-7.

Accepted: 25 February 2008 